DEPARTAMENTO DE PATOI.OGIA F: CIINICAS CIRÚRGICA E OIBSTETRICA Diretor: I’rof. Dr. Finesto Antonio Matera

\title{
CONTRIBUIÇÃO PARA O ESTUDO DA ANESTESIA DENTÁRIA DO CÃO \\ Via de acesso transorbitália à fossa pterigopalatina
}

(DF:NTAL ANAFSTIII:SIA IN THE DOG

Injection into the pterygo-palatine fossa by transorbital method)

\author{
J. S. Mikcontris Veicid \\ Instrutor
}

O tratamento das afeçōes dentárias do cāo, principalmente extraçōes de dentes, impõe, como norma preliminar, o emprêgo da anestesia por fatôres técnicos e humanitários.

Vários processos de anestesia podem ser utilizados, porém, poucos estāo isentos de criticas desfavoráveis por apresentarem dificuldades técnicas ou riscos desnecessários.

A amestesia geral è usada e indicada por vários autores e tratadistas, dentre os quais destacamos GARBUTY (1938), RICHIMAN (1938), Secord (1941), Garcia Alronso (1941), Branier (1942), KAPLAN (1949)), GUARI) (1953), ANNIS (1965).

Esta anestesia, bastante difundida entre nós, principalmente com o advento de barbitúricos de açāo ultra rápida (KeIBßaUY e cols. 1967 ), e realmente cómoda para o profissional, mas, a nosso ver, nem sempre bem indicada. Isto por nāo considerarmos lógico, para simples extraçōes dentárias, submetermos nossos pacientes a riscos desnecessários.

A allestesia local, por infiltração, consiste na deposição da solução anestésica junto ao periósteo, à altura do ápice das raizes dentárias. O anestésico aí colocado difunde-se através do periósteo, alcançando as terminações nervosas dos alvéolos e dentes.

Esste lipo de anestesia, excepçāo feita à extração de um ou dois dentes incisivos, oferece desvantagens que o contra indicam como de cleiçāo na prática cirúrgica cotidiana.

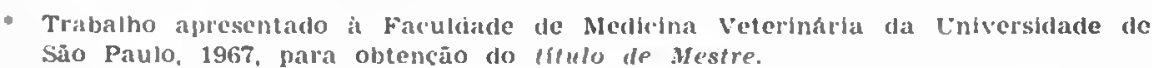


Pelos inconvenientes apresentados acima, os autores e clássicos da literatura cirúrgica veterinária iniciaram pesquisas, investigando vias de acesso a troncos nervosos, que permitam execuçāo fácil de uma anestesia regional capaz de propiciar completa insensibilização dentária. A solução anestésica colocada cm determinado ponto do trajeto nervoso, que vai do campo operatório aos centros nervosos superiores, bloqueia as transmissões dolorosas oriundas do campo distal.

A superioridade dêste tipo de anestesia, sôbre os demais, é confirmada pela dedicação dos estudiosos do assunto na investigação de boas vias de acesso, como observamos pelos trabalhos de Frank (1931), Bressot e Cliza (1931), Foust (1934), Lacrolx (1935-1953), Toso (1935), Haussler, Barth (cit. CiNoTTI - 1952).

Interessa-nos, mais objetivamente, as que dizem respeito às vias de acesso à fossa pterigopalatina.

Entre estas, verificamos que há manifesta preferência dos tratadistas, clássicos e autores, por uma ou outra das técnicas expostas.

A técnica de Frank (1931), ć accita por Wrighit (1952), Cinotet (1952), Westriues e Fristschi (1960), Lunib (1963) que a descrevem em scus tratados. De acôrdo com êstes autores, "para completo bloqueio do nervo infraorbitário, Frank desenvolveu método de injeção na fossa pterigopalatina, semelhante àquele adotado por Bemis, no cavalo, utilizando-se do espaço existente entre a borda posterior do osso maxilar e a borda coronoide da mandíbula".

Bressou e Cliza (1931) propōem outra via de acesso, que é a mesma ensinada por Garcis Alronso (1941). Indicam o canal infraorbitário, como a melhor via à fossa pterigopalatina do cão.

Foust (1934), propõe atingir o nervo maxilar, à sua saída do forame redondo, atravessando a mucosa do fundo do vestíbulo bucal, cêrca de $2 \mathrm{~cm}$ acima do último molar.

LACROIX (1953-1953), adota o seguinte plano para anestesia daquela fossa: "a agulha é introduzida pela face interna da arcada dentária, logo após e paralelamente ao último molar. A solução anestésica é injetada à medida que a agulha vai penetrando nos tecidos". Seu método é aceito por SCHNElle (1939) e por SECOD (1941), que o usam em suas intervençōes, indiferentemente, com a anestesia geral por barbitúricos.

Insatisfeitos com os métodos sugeridos pelos autores, por dificuldades técnicas de execução, iniciamos em 1955 estudos sôbre nova via de acesso que viesse sanar, tanto quanto possivel, as falhas e desvantagens observadas até cntão e satisfizesse os imperativos de facilidade técnica, comodidade, simplicidade ,eficiência, segurança e inocuidade. 
Nossos estudos levaram-nos a utilizar a via de acesso transorbitária á fossa pterigopalatina (MARCONDES VEIGA - 1956).

Com a progressão dos estudos e animados com os resultados, dedicamo-nos à pesquisa minuciosa da bibliografia especializada, quando então, encontramos o relato de Toso (1935) que descreve a via de acesso em aprêço, embora nāo apresente casuística de observações.

Continuamos a estudar e utilizar o método.

Os resultados obtidos, aliado ao aparente desconhecimento dos autores que, até o momento, continuam a fazer referéncias a processos considerados, por nós, deficientes e, por alguns insatisfatórios, animam-nos e justificam, a nosso ver, a apresentação dêste trabalho.

\section{SÜMULA ANATOMICA}

A porção oral da fossa pterigopalatina, que nos interessa no momento, acha-se limitada medialmente pelo segmento perpendicular do osso palatino, lateralmente pela extremidade oral do osso zigomática ou orbitária (Fig. 1) que se relaciona, dorso-medialpela tuberosidade do maxilar, que forma um assoalho ósseo.

Nesta região encontram-se vários forames: o palatino caudal e o esfenopalatino, intimamente relacionados e localizados na parede ântero-medial da fossa; o maxilar situado ântero-lateralmente aos dois primeiros e, caudalmente a êste, certo nümero de orificios para pequenos nervos e vasos destinados às raízes dos molares.

A fossa pterigopalatina é ocupada lateralmente pela glândula zigomática ou orbitária (Fig. 1) que se relaciona, dorso-medialmente, com a periórbita e medialmente com o músculo pterigoideo interno, artéria maxilar interna e nervos. Os espaço compreendido entre estas estruturas é preenchido por tecido adiposo.

Os nervos que atravessam esta região (Fig. 2), são originários do n. maxilar, que por sua vez é ramo do trigêmio. O n. maxilar subdivide-se em 3 ramos, dos quais apenas 2 nos interessam: o infraorbitário c o esfenopalatino.

O primeiro segue isolado até penetrar no canal homônimo e, antes de abandonar a fossa pterigopalatina, fornece ramos alveolares que, penetrando por pecjuenos orifícios existentes na tuberosidade maxilar, destinam-se aos molares. Já no canal infraorbitário, emite ramos alveolares que se constituem na principal inervação dos pré-molares, caninos e incisivos.

O segundo apresenta 3 ramos: o $n$. grande palatino ou palatino anterior, o n. nasal posterior e o n. palatino menor. Os dois primeiros individualizam-se junto a borda oral do músculo pterigoideo interno $e$ penetram respectivamente nos orificios palatino caudal e esfenopalatino. O n. grande palatino contribue, em parte, para a inervação dentária e gengival. 
Utilizamos para nossas verificações, animais encaminhados à exame clínico, ao Ambulatório de Cirurgia da Faculdade de Medicina Veterinária da Universidade de São Paulo e aos quais se impunha, como tratamento, extração de um ou vários dentes da arcada superior. Nossa casuística reune 152 anestesias, praticadas em 137 cães de sexos, raças e portes diversos, com idades variáveis entre 6 meses e 18 anos 16 animais de idades desconhecidas, 30 até 6 anos c 101 com 7 anos ou mais).

Adotamos para as anestesias dentárias, instrumental especializado (Fig. 3), evitando o aproveitamento de seringas e agulhas comuns. As seringas por nós usadas foram as chamadas "Carpule" ou similares. Estas, por sua conformação especial, permitem que as empunhemos com firmeza e exerçamos, concomitantemente, a pressão necessária à infiltração do anestésico nos tecidos, com a segurança que não nos possibilitaria uma seringa comum. Exclue-se por completo o perigo de rutura por excesso de pressāo ou movimentação brusca do animal. Suas agulhas, com 2,5 ou $3,5 \mathrm{~cm}$ de comprimento, conforme o intermediário utilizado, apresentam como principais características, grande resistência - embora flexiveis - aliada a pequeno calibre.

Não só devido ao uso de instrumental especializado, mas principalmente pelos resultados obtidos, aproveitamo-nos das soluções anestésicas associadas à drogas vasoconstrictoras, contidas nos chamados "Cartuchos" anestésicos. Éstes, com capacidade para 1,8 $\mathrm{ml}$, são encontrados com facilidade no comércio e preparados por diversos laboratórios farmacêuticos.

\section{ME'IODO}

O animal, devidamente amordaçado, era contido em decúbito lateral esquerdo ou direito, inversamente ao lado a anestesiar. Após antissepcia da pele, afastavamos, com o dedo, o globo ocular protegido pela pálpebra inferior, da borda dorsal da apófise zigomática do malar.

A altura do centro da fenda palpebral, introduziamos a agulha através da pálpebra, em sentido dorso-ventral, rente ao osso malar (Figs. 4 e 5a), até sentirmos que a mesma atingira o assoalho ćsseo, representado pela tuberosidade do maxilar. A medida que retiravamos a agulha, cêrca de meio centimetro, deixavamos $\mathrm{cm}$ seu trajeto mais ou menos um têrço do conteúdo da ampola.

Dando cm seguida, à agulha, inclinação aboral de aproximadamente 30 graus (Fig. $5 \mathrm{~b}$ ), penetravamos nos tecidos meio centimetro a mais que na manobra anterior, onde depositávamos a metade da solução anestésica restante no "cartucho". 
Repeliamos novamente a operação (Fig. 5c), dando entretanto à agulha inclinação de 45 graus c penetrando mais profundamente cêrea de meio centimetro.

Em todos êstes 3 tempos, a ponta da agulha avizinhava-se, sem toca-lo entretanto, do tronco nervoso que caminha através da fossa pterigopalatina.

As duas últimas manobras tinham por objetivo não só o envolvimento de maior segmento daquele tronco pela solução anestésica, mas, principalmente, o de bloquear os pequenos ramos nervosos que o abandonam, em direção à tuberosidade do maxilar, antes de sua penetração nos canais infraorbitário e esfenopalatino.

IRHSLILTADOS

Nossos resultados constam da Tabela I.

\section{DISCUSS $\pi O$}

As diversas técnicas de anestesias regionais, citadas na introdução do presente trabalho, merecem críticas desfavoráveis, as vêzes dos próprios autores que as transcrevem, devido quase sempre, à dificuldade de acesso à fossa pterigopalatina e cujos vicios passamos a descrever:

Assim, a técnica de FranK (1931) recebe de Wright (1952) a seguinte crítica: "o bloqueio do nervo maxilar, na fossa pterigopalatina, representa consideráveis dificuldades téenicas $e$ há incerteza de que o anestésico possa ser introduzido diretamente ao redor do nervo. Além disso, as manobras não são isentas de riscos". Acrescentemos apenas que esta técnica, pelas dificuldades que apresenta já deveria estar superada pelas descritas posteriormente, porém, trabalhos recentes (LuMis - 1963; Westhles e FritsciI 1960) aincla a ela fazem menções.

Quanto àquela proposta por Bressou e Cliza (1931), embora concordemos que o canal infraorbitário dê fácil acesso à região, acreditamos também, que a penetração da agulha por um conduto totalmente preenchiclo por nervos e vasos, que por êle transitam, é bastante perigosa pelos riscos que poderão advir para aquelas estruturas.

Estaríamos de pleno acôrdo com a técnica de LACRoIx (19351953), por ser a mais racional e anatômica das já descritas, não fôsse a necessidade de mantermos, durante a prática da anestesia, o animal com a bóca abcrta, sem reaçōes violentas por parte do mesmo. Isto pode ser conseguido praticando-se anestesia de base, ou melhor ainda, uma narcose, conforme aconselha, aliás, o autor. 
Esta narcose, entretanto, aproxima-se bastante de uma anestesia geral, que justamente é nosso objetivo evitar.

O método de Foust (1934) apresenta, aproximadamente, os mesmos vícios da técnica anterior.

As anestesias regionais ou de bloqueio devem merecer, realmente, a preferência dos profissionais, pelas vantagens que podem oferecer:

a) não apresentam os riscos inerentes às anestesias gerais;

b) oferecem grande área anestesiada, com um minimo de picadas de agulha;

c) exigem menor volume de soluçāo anestésica;

d) permitem trabalho fora do campo operatório, o qual, quase sempre, apresenta afecções que contra indicam injeções locais.

Contudo, o estudo da anestesiologia, particularmente o das classificadas como anestesias regionais, nos demonstra que de maneira alguma estamos livres de insucessos. Analizando entretanto as origens destas falhas, descobrimos que algumas das suas causas não são, em geral, inerentes ao caso em si, mas, quase sempre, devidas a técnicas defeituosas.

Não nos podemos furtar, nesta altura, de transcrever os ensinamentos de DogliotTi (1943), que afirma: "o anestesista deverá recordar com exatidão a conformação do esqueleto, muito freqüentemente a base dos necessários pontos de reparo e de orientação para uma boa anestesia periférica".

A confirmação destas categorizadas palavras, encontramos no Compêndio de Anestesia Dentária de Nevis̄ e PuTrerrbaug (1949): "nāo é bastante o conhecimento do ponto de introdução da agulha e a direçāo a dar a esta. Necessário se torna um razoável conhecimento anatômico da região, a fim de fugirmos a um empirismo que deixaria o anestesista embaraçado com aparecimento de qualquer complicação".

Algumas causas de malogro seriam devido:

a) variaçōes na estrutura e formas ósseas, nāo só de animal para animal, mas, principalmente, de raça para raça;

b) desconhecimento anatômico da regiăo e inexperiência do profissional no uso dêste tipo de anestesia;

c) inicio precoce do ato operatório, antes da anestesia estar perfeitamente instalada.

No que se refere ao nosso estudo e ao item a, observamos pela casuística (Tabela I), que praticamos a anestesia em animais de 
portes e raças diversas sem o menor fracasso. Isto porque, as diferenças de conformação da cabeça não repercutem, com a mesma intensidade, na região abordada.

Quanto ao item $b$, os pontos anatômicos de referências (centro da fenda palpebral, borda dorsal da apófise zigomática do malar e assoalho ósseo) são fàcilmente identificáveis, o que simplifica, sobremodo, a técnica. A anestesia em aprêço, vem sendo utilizada há mais de 10 anos e pudemos verificar que alunos e profissionais, a quem tivemos oportunidade de ensinar a técnica, foram capazes de realiza-la com sucesso, após ligeira explicação e demonstração, sem exigência de prévia experimentaçāo.

Quanto ao item $c$, êste nāo revela propriamente deficiência técnica em si, mas impaciência do profissional em dar início ao ato operatório. Em verdade, parte do "êxito das anestesias parciais, está no saber esperar" (CRISTMANN e cols. 1948). MinitT e col. (1951) considerando que "15 minutos são longos para se esperar" - ou, "os mais longos", no dizer de outro mestre - aconselham comprovação pelo relógio.

No inicio de nossos estudos, aguardavamos cêrca de $10 \mathrm{minu}$ tos antes de iniciarmos as extraçooes dentárias. Posteriormente, com a rotina de serviço, o tempo de espera variou de 10 a $20 \mathrm{mi}$ nutos, por nảo considerarmos fator de importância para comprovação do método. Antes das extraçōes, a instalaçāo da anestesia era testada à altura da gengiva, comprimindo-a com a espatula. Um sinal observado, com certa freqüência, era o abrir e fechar da boca do animal, seguido de movimentos com a pata, como se o mesmo procurasse retirar um corpo estranho localizado entre os dentes.

Outro ponto que merece comentários é quanto ao tecido a ser penetrado pela agulha.

Toso (1935), faz referência tôda especial, e considera preferivel, a introdução da agulha através do fornice conjuntival lembrando, contudo, que por excesso de prudência, pode-se atingir o mesmo objetivo por via transcutânea.

Esta última, foi a preferida por nós, por motivos que reputamos razoável: devem sempre merecer primazia os processos seguros, em detrimento daqueles mais temerários, desde que o objetivo seja alcançado da mesma forma.

Aquele Autor, introdu\% a agulha no limite entre o têrço médio e medial da fenda palpebral, dando à mesma inclinaçāo aboral e medial, em direçāo ao tronco nervoso, aconselhando aprofunda-la cêrca de 2 centímetros. Esta última indicação, não muito precisa - pois na prática clinica encontramo-nos frente a animais de diversos portes - exige cuidados especiais e experiência por parte 
do profissional, a fim de que a agulha não traumatize o tronco nervoso e vasos satélites.

A introdução da agulha - como fizemos em nossas anestesias - perpendicularmente e junto à apófise zigomática do malar até atingir o assoalho ósseo (tuberosidade do osso maxilar), oferece, a nosso ver, referências anatomicas mais precisas; além do mais a agulha não caminha $\mathrm{em}$ direção ao tronco norvoso, mas, sim, lateralmente ao mesmo, afastando-se o risco de lesá-lo.

Poderiamos aventar a hipótese de que, junto a apófise zigomática, a glândula homônima possa ser eventualmente atingida, principalmente, quando damos à agulha inclinaçōes mais abolais. Em nossas anestesias, porém, não observamos sinais de lesão glandular, que determinasse quaisquer complicaçōes imediatas ou tardias.

Quanto ao comprimento da agulha, lambém não acompanhamos a indicação de Toso (1935); encuanto o autor aconselha uso de agulhas de 4-6 $\mathrm{cm}$, praticamos nossas anestesias com agulhas de 2,5-3,5 cm de comprimento.

Embora, aparentemente, sem importância, êste pormenor reveste-se de certo valor. A utilização de agulhas relativamente longas, implicaria no uso obrigatório de scringas $\mathrm{e}$ agulhas hipodérmicas comuns, afastando, por completo, o aproveitamento de instrumental especializado, cujas agulhas tîm comprimento máximo, util, de $3,5 \mathrm{~cm}$.

Quanto à solução anestésica empregada, levamos em conta apenas o volume injetado. Não consideramos o sal anestésico nem sua concentração, por tratar-se de estudo relacionado à técnica e não à eficiência maior ou menor dêste ou ałucle anestésico.

Toso (1935) indica volume variável de $2-3 \mathrm{ml}$. Tivemos cuidado, para atender padronização, de aplicarmos sistemàticamente $1,8 \mathrm{ml}$ de solução, que é o volume contido nos "eartuchos" anestésicos, se bem que, injeção de volume maior não viesse invalidar o método.

\section{CONCILISOLS}

O estudo da via de acesso transorbitária, sua utilizacāo na prática corrente e a casuística das obscrvaçōes, permite-nos concluir que:

1 - a via de acesso transorbitária permite a prática da anestesia com o animal amordaçado, o que representa indiscutivel segurança para o profissional;

2 - é de fácil execução o que possibilita, mesmo à iniciantes e inexperientes, obtenção de completa anestesia da arcada dentária superior, nāo constituindo óbice as diferenças anatômicas de raças; 
3 - a penetração da agulha se faz em tecidos sem importância anatômica (pele e tecido adiposo), e que atingidos, como é óbvio, não acarretam qualquer pertubação ao animal. Não se observaram alteraçōes imediatas ou tardias que sugerissem lesões da glândula zigomática ou estruturas vizinhas;

4 - a introdução da agulha através da pele é preferivel, pois a palpebra, além de oferecer proteção segura ao globo ocular, na eventualidade de movimentação inesperada do animal, impede sua visão, prevenindo reações desfavoráveis de defesa;

5 - atingimos fàcilmente as proximidades do tronco nervoso, sem perigo de lesá-lo, pois a agulha, no ponto indicado passa lateralmente ao mesmo;

6 - não há desperdicio de solução anestésica, pois esta é injetada próxima ao tronco nervoso;

7 - peq̨ueno volume de solução $(1,8 \mathrm{ml})$ foi suficiente para obtençāo de anestesia completa da hemi arcada superior, não influindo o porte do animal;

8 - a utilização de instrumental especializado é viável, pois agulhas de $2,5-3,5 \mathrm{~cm}$ de comprimento foram suficientes para execução da anestesia $\mathrm{cm}$ animais de qualquer porte;

9 - foi praticada indiferentemente em animais jovens e idosos, sem complicações de qualquer natureza, trans ou pós anestésicas;

10 - tôdas as anestesias coroaram-se de êxito, permitindo extrações imediatas de, as vézes, vários dentes de hemi arcada superior.

\section{SUMMARY}

The regional anaesthesia in the dog, for dental block is studied by the Author:

Methods of injections into the pterygo-palatine fossa are described, receiving attention the Toso's technique, with some modifications.

Anatomical aspects, instruments and technique applied ar present, so the Author's conclusion.

\section{REI'FRENCIAS BIBLIOGRAFICAS}

ANNIS. J. R. - Teeth. In: ARCHIBALI), J. - Canine surgery: a text and referecence work. First Archibald edition. Wheaton, American Veterinary Publications, Inc., 1965, p. 329-344. 
RRAMFiR, C. N. - Dentistry as applied in small animal pratice. N. Amer. Vet., $2 S(7): 466-469,1942$.

BRFSSOU, C. \& CLIZA, S. - Contribution à l'étude de l'anesthésie dentaire chez le cheval et chez le chien. Rev:. Méd. vét., Alfort, 107(3):129-134, 1931.

CHRISTTMANN, F. F..; OTTOLFNGHI, C. E.; RAFFO, J. M.; GROLMAN, G. - Técnica cirürgica. v. 1. Rio de Janeiro, kiditóra Guanabara, 1948, p. 160.

CINOTTI, F. - Medicina operatoria veterinaria. Milano, Francesco Vallardi, 1952 , p. $136-140$.

DOGL,IOTTI, A. M. - Tratado de anestesia. Rio de Janeiro, Editóra Guanabara, 1943, p. 283-296.

ELLFMBF:RGF:R, W. \& BAUM, H. - Anatomie descriptive et topographique du chien. Paris, Reinwald \& Cie, Libraires-Editeurs, 1894.

FOUST, H. L. - The anatomy of the regions concerned in a method of dental nerve blocking in the dog. N. Amer. Vet., 15(4):41-43, 1934.

FRANK, F:. R. - Dental anesthesia in the dog. Vet. Med., 26(11):462-463, 1931.

GARBUTT, R. J. - Diseases and surgery of the dog. New York, Orange Judd Publishing Company Inc., 1938, p. 31.

GARCIA ALFONSO, C. - Operaciones en veterinaria. Madrid, Imprenta Biosca, 1941, p. 421-431.

GUARD, W. F. - Surgical principles and technics. Ohio, published by the author, 1953, p. 191-193.

KAPI,AN, A. D. - Extration of tecth in small animals. Vel. Med., 44(8): 355-357, 1949.

KFIRBAUY, A. M. R. M.; KFRBAUY, V. F. C. J.; MARCONDHS VFigA, J. S. - Anestesia geral de pequenos animais com o emprégo do Metorxital-Sódico (Brietal Sódico). Rev. Med. Vet., S. Paulo 2(3):196202, 1967.

LACROIX, J. V. - Canine dental surgery: its indication and anesthesia. Cornell Vet., 25(3):247-258, 1935.

LACROIX. J. V. - Canine surgery. 3rd ed. Evanston, American Veterinary Publications, Inc., 1953, p. 242-244.

LTMR, W. V. - Small animal anesthesia. Philadelphia, Lea \& Febiger, 1963, 1.) $256-257$.

MARCONDFS VFIGA, J. S. - Nota preliminar sôbre o estudo da anestesia dentária do cão. Rev. Fuc. Med. vet., S. Paulo, 5(4):653-661, 1956.

NF.VIN, M. \& PUTTERBACGi, P. G. -.- Anestesia dentária. Rio de Janeiro, Fditôra Cientifica, 1949, p. 35, 337-347.

RICHMAN, S. - Flap operation for the removal of the canine tooth. $N$. Amer. Vel, $19(12): 64-66,1938$. 
SECORD, A. C. Small animal dentistry. J. Amer, vet. med. Ass., 98(771) : $470-476,1941$.

SCHENELLE, G. B. - Some observation on dental diseases in the dog. $N$. Amer. Vet., 2o(8):42-45, 1939.

TOSO, G. - Contributto all'anestesia dentale nel cane. Separata Nuovo Ercolani, 13(4):2-15, 1935.

WESTHUFS, M. \& FRITSCH, R. - Die Narcose der tiere. v. 1. Berlim, Paul Parey, 1960, p. 80-81.

WRIGHT, J. G. - Veterinary anesthesia. 3rd ed. London, Baillière, Tindall \& Cox, 1952, p. $47-50$. 


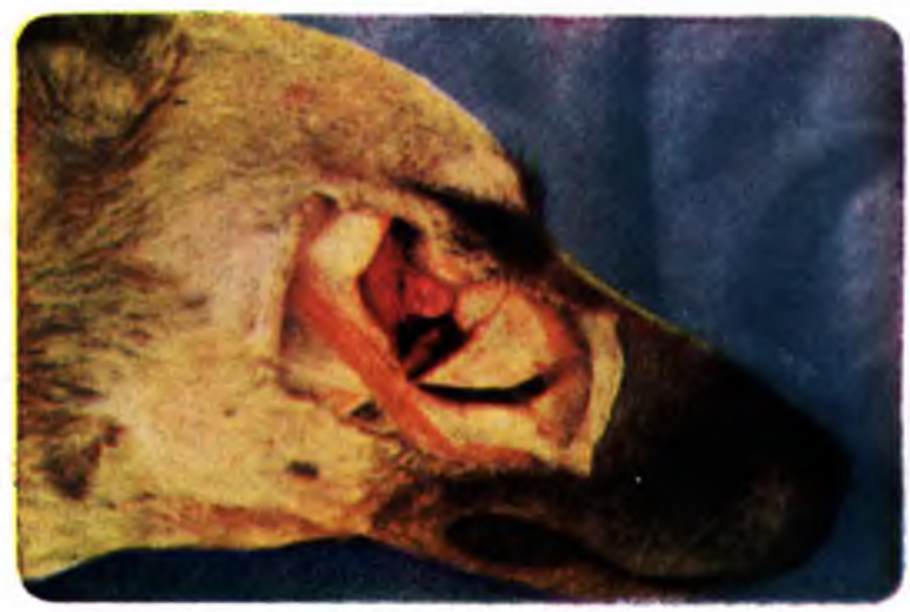

Fig. 1

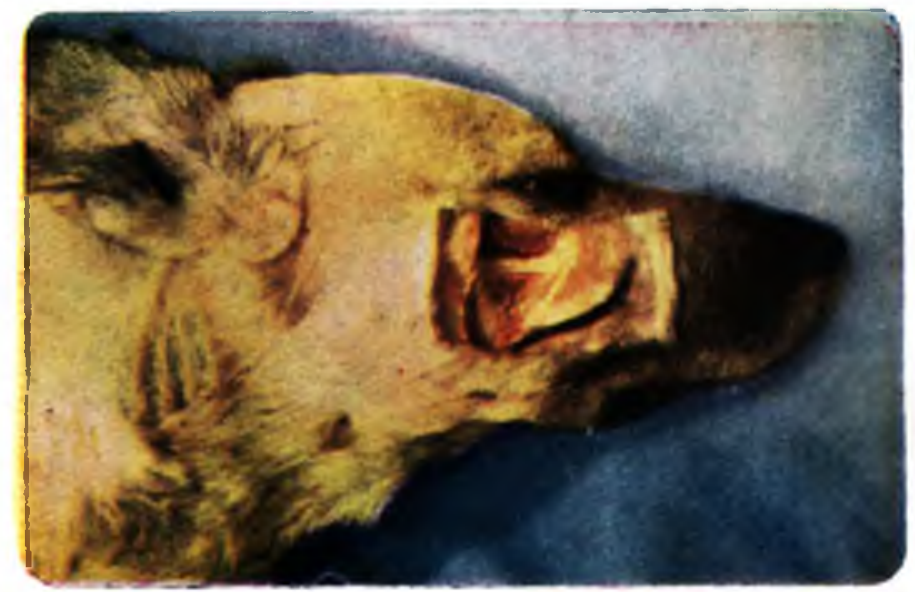

Fig. 2

Fig. 1 - a) gl. zigomática; b) periórbita; c) tec. adliposo.

Fig. 2 - d) n. infraorbltárjo; e) n. esfenoplatino; f) ramos alveolares. 

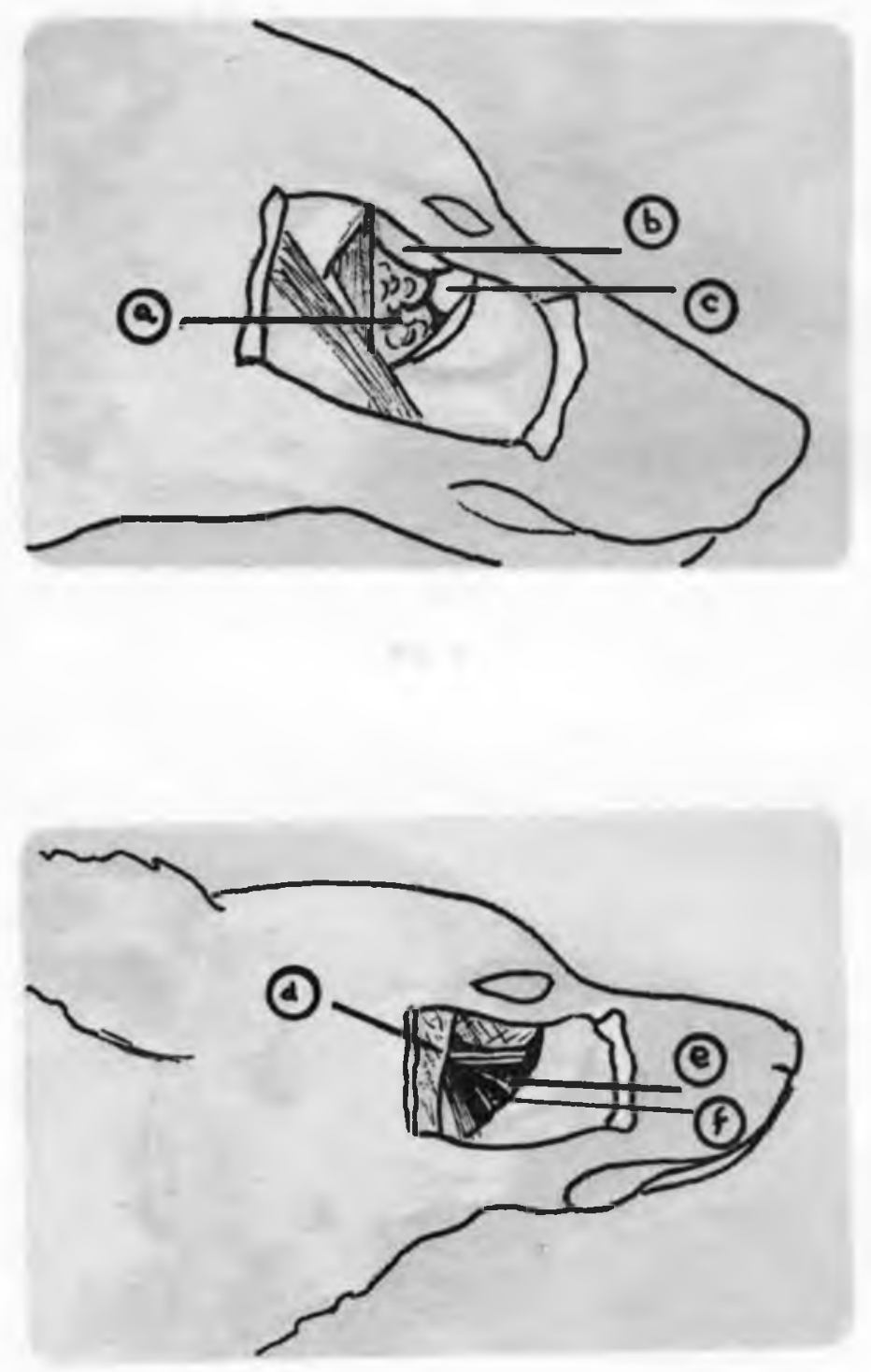


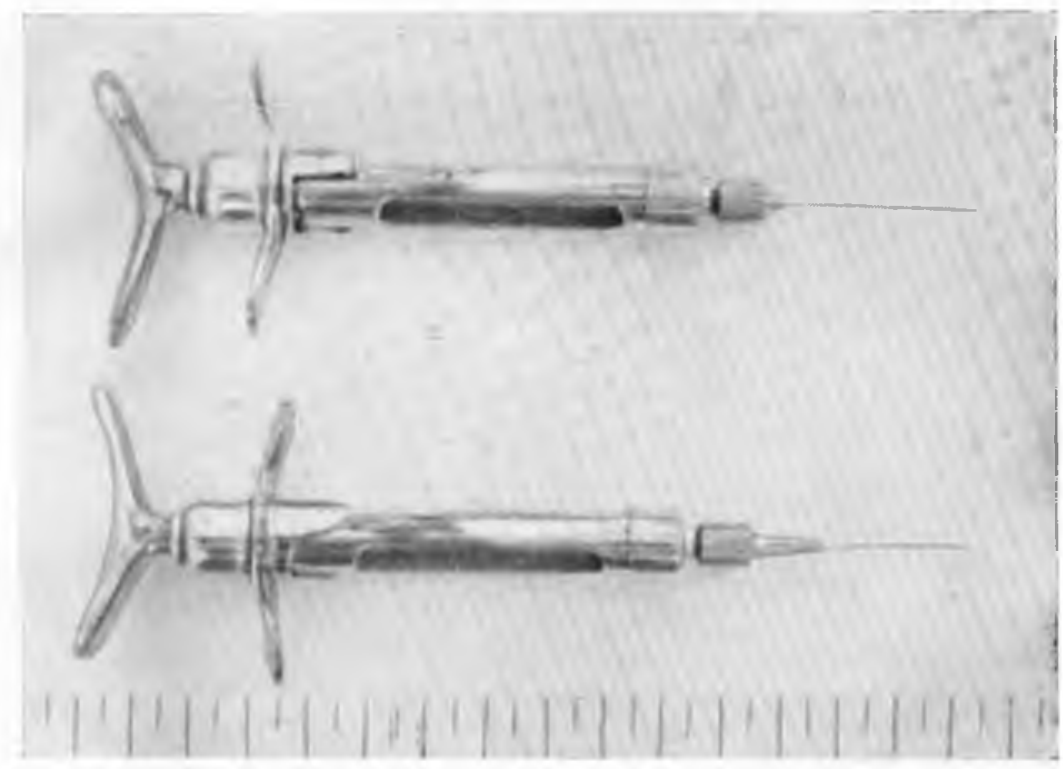

Fig. 3 - Instrumental

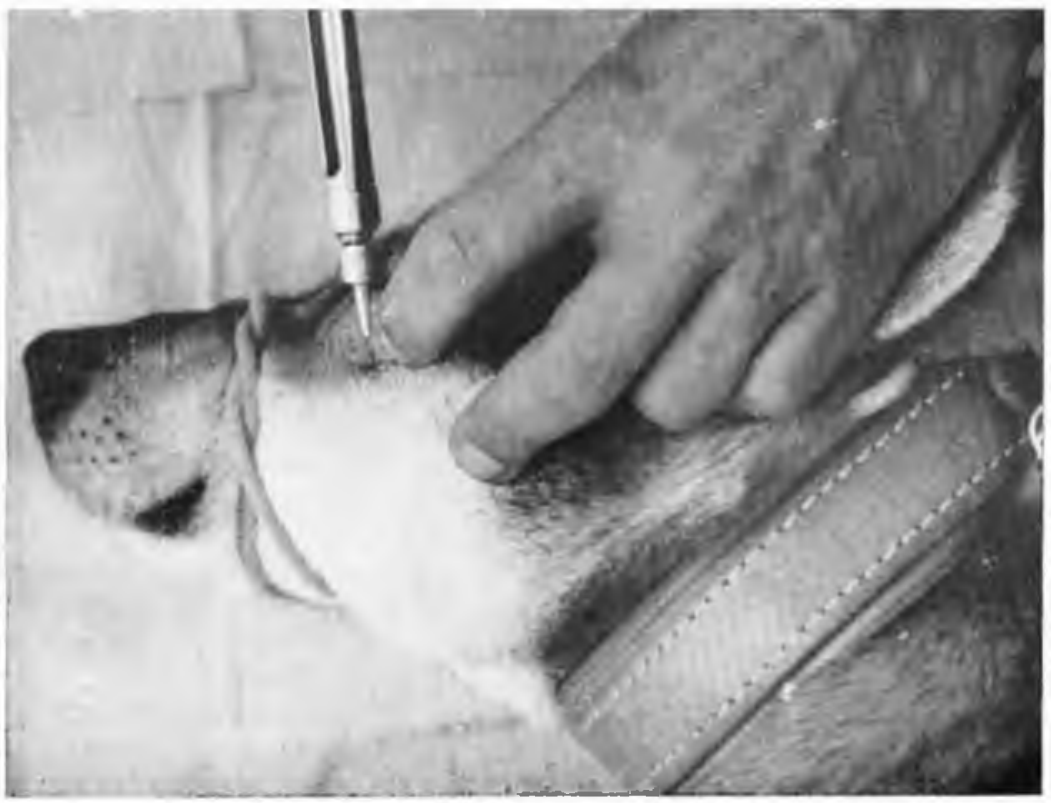

Fig. 4 - Ponto de introducão da agulha. 


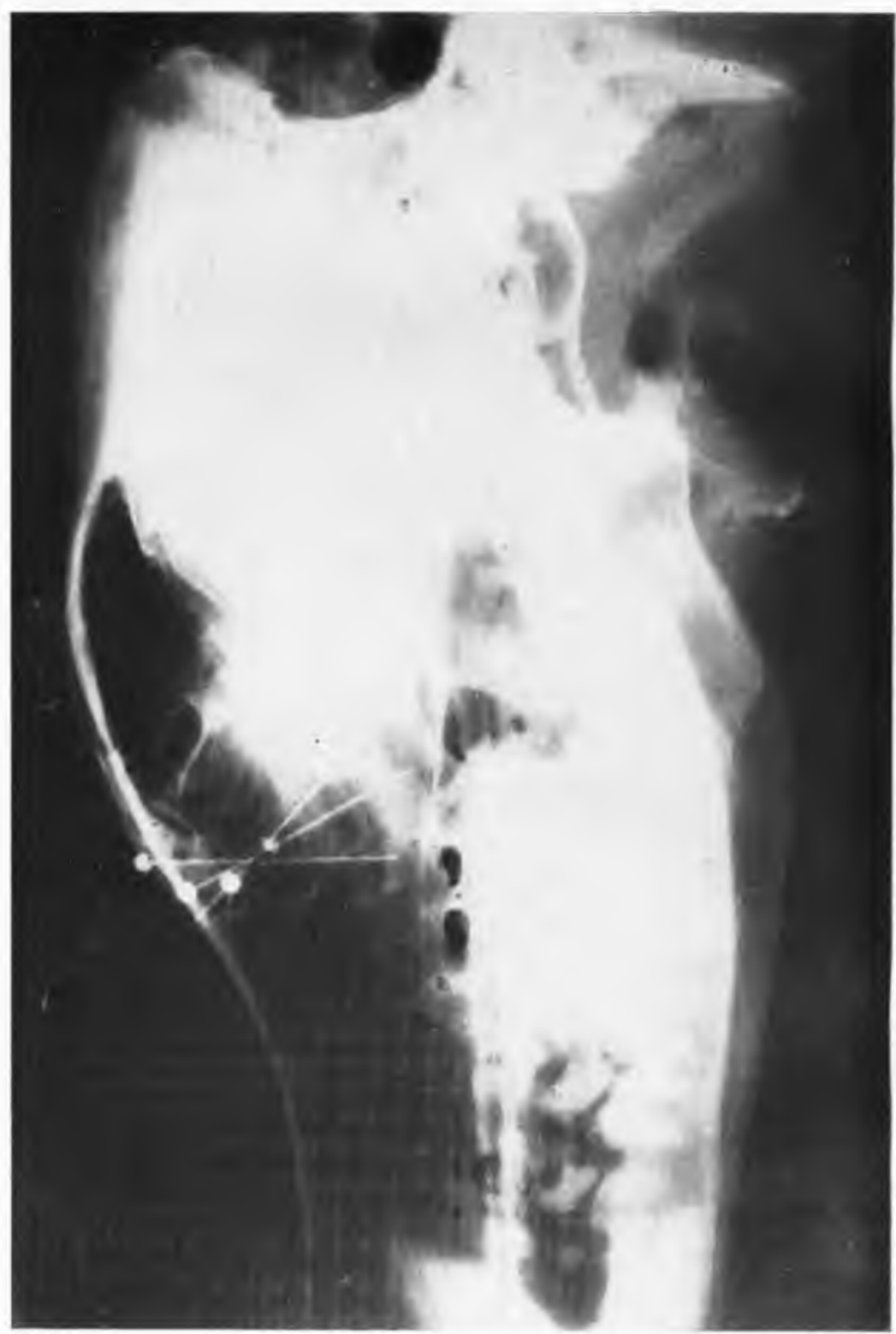

Fig. 5 - Aspecto radiológico das posicōes da agulha. 


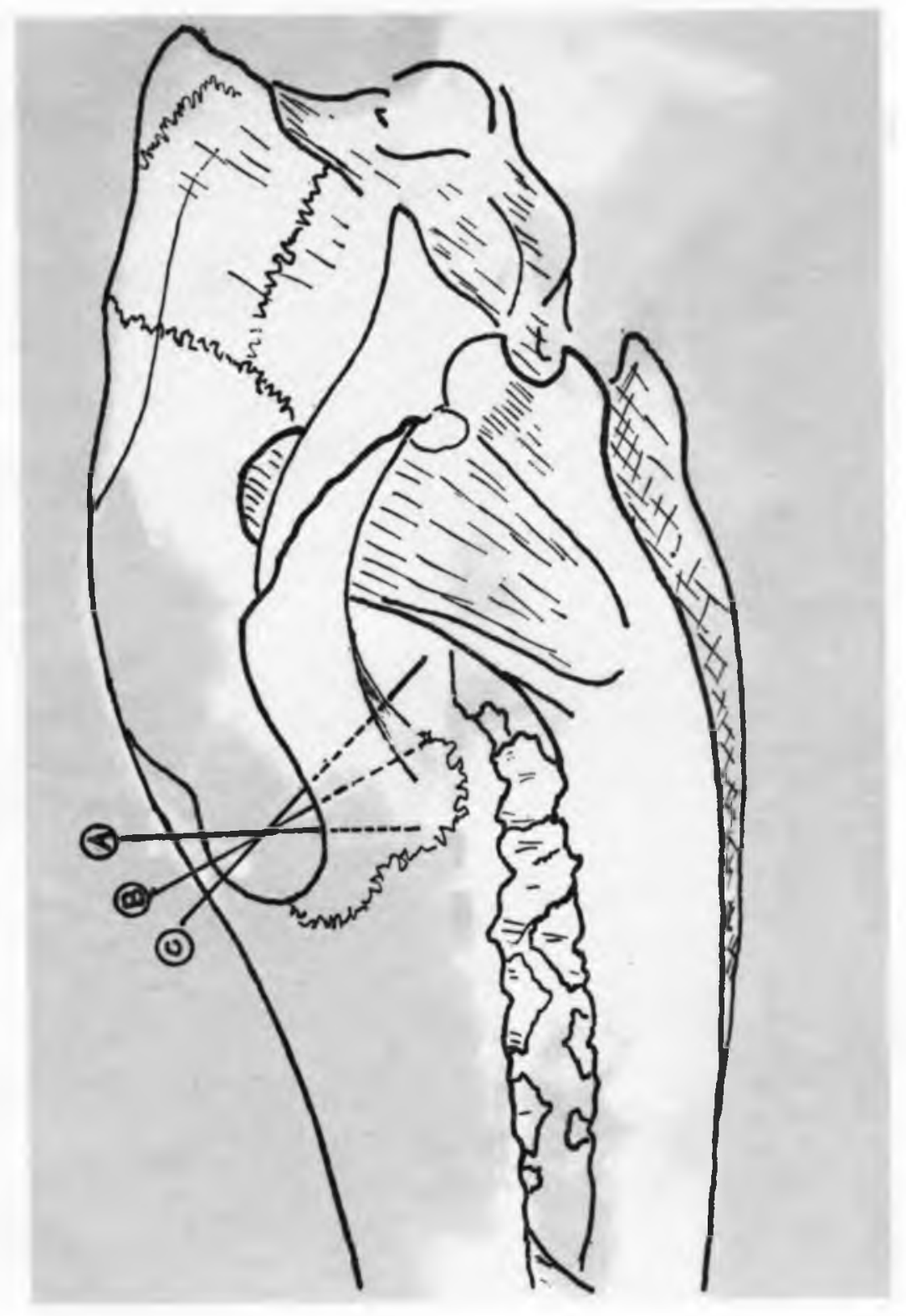


TABELA I

\begin{tabular}{|c|c|c|c|c|c|}
\hline Obs. & $\begin{array}{c}\text { Regis- } \\
\text { tro }\end{array}$ & Raca & Idade & Dentes extraidos & Resultado \\
\hline 1 & - & s.r.d. & $13 \mathrm{a}$ & $1 . \circ 2.0^{\circ} \mathrm{I}$ & Bom \\
\hline 2 & $\longrightarrow$ & s.r.d. & 9 a & $2.0^{\circ} 3 . \circ \mathrm{I}, \mathrm{C}, 1.02 .0 \mathrm{MI}$ (E) & Bom \\
\hline 3 & 210 & c.s. Fox & $6 a$ & 4.0 PMI & Bom \\
\hline 4 & 393 & Basset & $15 \mathrm{a}$ & $C(E)$ & Bom \\
\hline 5 & 393 & Basset & $15 \mathrm{a}$ & C (D) & Bom \\
\hline 6 & 439 & s.r.d. & $5 \mathrm{a}$ & C (E) & Bom \\
\hline 7 & 514 & s.r.d. & 9 a & $1.0^{\circ} 2 .^{\circ} 3 . \circ 4.8 \mathrm{PM} \quad(\mathrm{D})$ & Bom \\
\hline 8 & 514 & s.r.d. & 9 a & C, $1.0^{\circ} 2.0^{\circ} 3.0^{\circ} \mathrm{PM}$ (E), $1.0^{\circ} 2 . \mathrm{M}^{\circ}$ (D) & Bom \\
\hline 9 & 770 & c.s. Fox & $9 \mathbf{a}$ & $1.0 \mathrm{M}$ & Bom \\
\hline 10 & $\$ 99$ & Basset & $6 a$ & 4.9 PM & Bom \\
\hline 11 & 907 & s.r.d. & $8 \mathrm{a}$ & 1.० PM (E) & 13om \\
\hline 12 & 929 & Basset & $7 a$ & 1.० 4.० PM, $1 . \circ \mathrm{MI}$ & Bom \\
\hline 13 & 1045 & c.s. Pastor & $10 \mathrm{a}$ & C (F) & Bom \\
\hline 14 & 1053 & Basset & $7 \mathbf{a}$ & 1.० PM (E) & Bom \\
\hline 15 & 1089 & s.r.d. & $7 \mathbf{a}$ & 4.० PM (E) & Bom \\
\hline 16 & 1746 & s.r.d. & 16 a & 1.० I, C, 4.0 PM (E) & Bom \\
\hline 17 & 1746 & s.r.d. & $16 \mathrm{a}$ & C, $1.01 .0 \mathrm{PM}$ (D) & Bom \\
\hline 18 & 1907 & s.r.d. & 8 a & $1 . " 2.03 .0 \mathrm{I}, 2.03 .0 \mathrm{PM}, 1.0 \mathrm{M}$ (E) & Bom \\
\hline 19 & 2457 & Fox & $11 \mathrm{a}$ & 3." I, C, 4.0 PM, 1.० M & Bom \\
\hline 20 & 2768 & Fox & $8 a$ & 4.0 PM (E) & Bom \\
\hline 21 & 3945 & Lúlú & $12 \mathrm{a}$ & 4. PM, $1.0 \mathrm{M}$ (E) & Bom \\
\hline 22 & 3989 & Lúlú & $7 a$ & C, $30^{\circ} 4 . \circ \mathrm{PMI}$ (D) & som \\
\hline 23 & $439-56$ & s.r.d. & 5 a & C (E) & Bom \\
\hline 24 & 770 & c.s. Fox & $9 a$ & $1.0 \mathrm{M}(\mathrm{E})$ & Bom \\
\hline 25 & 899 & Basset & 6 a & 4.0 PM (D) & Bom \\
\hline 26 & $90^{7}$ & s.r.d. & $8 a$ & $1 . \circ \mathrm{PM}(\mathrm{E})$ & Bom \\
\hline 27 & 929 & Basset & 7 a & $\mathrm{M}(\mathrm{E})$ & Bom \\
\hline 28 & 1045 & c.s. Pastor & 70 a & $C(E)$ & Bom \\
\hline 29 & 1159 & s.r.d. & $\bar{a}$ & 4.० PM (D) & Bom \\
\hline 30 & 1716 & s.r.d. & 16 a & 1.॰ I, C, 4.॰ PM (E) & Bom \\
\hline 31 & 1746 & s.r.d. & 16 a & $1.04 .0 \mathrm{PM} \mathrm{(D)}$ & Bom \\
\hline 32 & 1907 & s.r.d. & 8 a & $1.02 .03 . \circ \mathrm{I}, 2.03 .0 \mathrm{PM}, 1.0 \mathrm{M}$ & Bom \\
\hline 33 & 2457 & Fox & $11 \mathrm{a}$ & $3.0 \mathrm{I}, 40^{\circ} \mathrm{PM}, 1.0^{\circ} \mathrm{M}$ (D) & Bom \\
\hline 34 & 2768 & c.s. Fox & 8 a & 4.० PM (E) & .8om \\
\hline 35 & ऑs1 & r.s. Lúlú & $13 a$ & $3 .^{\circ} 4 .^{\circ} \mathrm{PM}, 1.0^{\circ} \mathrm{M}$ (D) & Bom \\
\hline 36 & 3989 & c.s. Lúlú & $7 \mathbf{a}$ & C, 3.० 4.0 PM (D) & Bom \\
\hline 37 & $540-57$ & s.r.d. & $11 \mathrm{a}$ & $3.04 .0 \mathrm{PM}, 1.0 \mathrm{M}$ (D) & Bom \\
\hline 38 & 540 & s.r.a. & 11 a & $20^{\circ} 3 . \circ 4.0 \mathrm{PM}, 1.0^{\circ} 20^{\circ} \mathrm{M} .(\mathrm{E})$ & Bom \\
\hline 39 & S84 & - & $14 \mathrm{a}$ & $3 .^{\circ} 4 . \circ \mathrm{PM}(\mathrm{E})$ & Bom \\
\hline 40 & 1117 & s.r.d. & $10 \mathrm{a}$ & $1.02 .0 \mathrm{PM}$ (E) & Bom \\
\hline 41 & 1565 & s.r.d. & $10 \mathrm{a}$ & $C$ (D) & Bom \\
\hline 42 & 2066 & Fox & 9 a & 4. PM (D) & Bom \\
\hline 43 & 2153 & s.r.d. & $5 \mathbf{a}$ & 4. PNI (D) & Bom \\
\hline 44 & 2525 & s.r.d. & $15 \mathrm{a}$ & C (E), $1.0 \mathrm{PM}$ (D) & Hom \\
\hline 45 & 2803 & Fox & $8 \mathbf{a}$ & 1.० PM (D) & Bom \\
\hline 46 & $311 \mathrm{j}$ & s.r.u. & 8 a & $2.03 .04 .0 \mathrm{PM}(\mathrm{C})$ & Bnm \\
\hline 47 & $3+114$ & Doberman & - & 4.0 PMI (D) & Bom \\
\hline 48 & -1027 & s.r.d. & $8 a$ & 3.० 4.0 PM, $1.02 .0 \mathrm{M}$ (D) & Bom \\
\hline $\begin{array}{l}\text { c.s. } \\
\text { a } \\
\text { s.r.d. }\end{array}$ & $\begin{array}{l}\text { - com } \\
\text { - anos } \\
\text { - sem }\end{array}$ & $\begin{array}{l}\text { sangue } \\
\text { raca definida }\end{array}$ & $\begin{array}{l}\mathrm{PMI} \\
\mathrm{I}\end{array}$ & $\begin{array}{l}\mathrm{M} \text { - molares } \\
\text { (D) - direlto } \\
\text { (E) - esquerdo }\end{array}$ & \\
\hline
\end{tabular}


TABELA I -- (Continuas̆o)

\begin{tabular}{|c|c|c|c|c|c|c|}
\hline Obs. & $\begin{array}{c}\text { Regis - } \\
\text { tro }\end{array}$ & Raça & Ida & de & extraldos & lresultado \\
\hline 49 & 4135 & Basset & 7 & $\mathbf{a}$ & 1."PM ( $\mathbf{P})$ & I3om \\
\hline 50 & 4352 & s.r.d. & 7 & $\mathrm{a}$ & $30^{\circ} 40^{\circ} \mathrm{PM}, 10^{\circ} 20^{\circ} \mathrm{MI}$ (F) & $130 \mathrm{~m}$ \\
\hline 51 & 4580 & s.r.el. & 12 & $\mathbf{a}$ & $2.01,1.02 .{ }^{\circ} \mathrm{PM}$ (D) & I3om \\
\hline 52 & $4 \times 65$ & s.r.d. & - & & $C:(E)$ & $130 \mathrm{~m}$ \\
\hline 53 & $127-58$ & s.r.d. & 8 & a & 2." $\mathrm{MI}(\mathrm{I})$ & Isom \\
\hline 54 & 288 & s.r.d. & 8 & $\mathbf{a}$ & $1.02 .0 \mathrm{M}(\mathrm{I}))$ & $130 \mathrm{~m}$ \\
\hline 55 & 288 & s.r.d. & 8 & a & $2.0 \mathrm{MI}(\mathrm{I})$ & I30m \\
\hline 56 & $6: 35$ & $\cdots$ & 7 & a & $3.0 \mathrm{I}(\mathrm{E})$ & liom \\
\hline 57 & $17: 32$ & Pequinès & 6 & a & 4.0 PM (D) & $130 \mathrm{~m}$ \\
\hline 58 & 3361 & s.r.d. & 9 & al & 2.01 & I30m \\
\hline $5 !$ & 3392 & s.r.a. & 7 & $a$ & $4.0 \mathrm{PM}(\mathrm{E})$ & $130 \mathrm{~m}$ \\
\hline 60 & 1826 & s.r.d. & 1.3 & a & $1.02 .03 .04 .0 \mathrm{PMI}, 1.0 \mathrm{M}$ (E) & $13 u m$ \\
\hline (\$1 & 4826 & s.t.d. & 13 & a & $1.02 .03 .04 .0 \mathrm{PMI}, 1 . \circ \mathrm{M}$ (D) & $130 \mathrm{~m}$ \\
\hline (i2 & $94-59$ & s.r.r. & 12 & $\mathbf{a}$ & $\mathrm{C}(\mathrm{D})$ & $130 \mathrm{~m}$ \\
\hline 63 & 117 & s.d.d. & 11 & a & 4.") PMI (1:) & I30m \\
\hline 6 & 647 & Pequinēs & 10 & a & 4." PMI ( $\mathrm{E})$ & $130 \mathrm{~m}$ \\
\hline (ii) & 647 & Pcquinés & 10 & $\mathbf{a}$ & 4." I'M (D) & Bom \\
\hline 66 & 1676 & s.r.d. & 13 & a & 1.0 IPI. 1.0 Mt ( $\mathrm{E})$ & $130 \mathrm{~m}$ \\
\hline 67 & 1676 & $\sin \cdot \mathrm{d}$ & 13 & a & $1 . " 2.53 .04 .0 \mathrm{I} \mathrm{M}, 1.02 .0 \mathrm{MI}$ (I) & Hom \\
\hline 68 & 2158 & Poodle & 6 & $a$ & $4 .^{\circ} \mathrm{PM}$ & I30m \\
\hline 69 & 2810 & c.s. Fox & 10 & a & 4." PM (E) & $130 \mathrm{~m}$ \\
\hline 70 & 3073 & s.r.d. & 9 & a & C., 4.० PMI (F) & I3om \\
\hline 71 & 3073 & s.r.t. & 9 & $\mathbf{a}$ & $2 .^{\circ} 3 .^{\circ} \Gamma \mathrm{M}, 1 .{ }^{\circ} 2.0 \mathrm{M}$ (D) & I3om \\
\hline 72 & 3328 & s.r.d. & - & & C, 1.02 .0 PXI (E) & Bom \\
\hline 73 & 3702 & c.s. Fox & 10 & $\mathbf{a}$ & 4." PMI (F) & $130 \mathrm{~m}$ \\
\hline 74 & 3807 & s.r.d. & 15 & a & 4." PM (E) & I3om \\
\hline 75 & 4152 & s.r.r. & 10 & a & 4.0 IMI (E) & Bom \\
\hline 76 & 4786 & s.i.a. & 11 & $\mathbf{a}$ & 4.0131 & $130 \mathrm{~m}$ \\
\hline 77 & 4938 & s.r.d. & 13 & $\mathbf{a}$ & $1.2131(F)$ & $130 \mathrm{~m}$ \\
\hline 78 & $428-60$ & & 8 & $\mathrm{a}$ & 1.0 I'M (D) & $130 \mathrm{~m}$ \\
\hline 79 & 8()$, & Pequincs & 5 & $a$ & 4. $P M(F)$ & $130 \mathrm{~m}$ \\
\hline 80) & 1347 & Pastor & 1 & a & $44^{\circ}[3 M(\mathrm{E})$ & $130 \mathrm{~m}$ \\
\hline 81 & 1531 & s.r.d. & 9 & $\mathbf{a}$ & $4.013,1 \% 1$ & $130 \mathrm{~m}$ \\
\hline 82 & 15.31 & s.r.cl. & 9 & $\mathbf{a}$ & 2.040 IPM (H) & $130 \mathrm{~m}$ \\
\hline 83 & 2546 & Doberman & 5 & a & 4.० PM (I)) & $130 \mathrm{~m}$ \\
\hline 84 & 2852 & s.r.d. & 9 & $\mathbf{a}$ & $1.0 \mathrm{PM}(\mathrm{D})$ & Hom \\
\hline 85 & 2971 & s. $d^{*} \cdot d$ & 12 & a & $1.02 .0 \mathrm{PM}, 1.0 \mathrm{Ml}(\mathrm{E})$ & Bom \\
\hline 86 & 4709 & - & 14 & $\mathbf{a}$ & $1.0 \mathrm{M}(\mathrm{D})$ & $130 \mathrm{~m}$ \\
\hline 87 & 5178 & s.r.di. & 9 & $a$ & 4. $\mathrm{I} M \mathrm{MI}(\mathrm{I})$ & Bom \\
\hline 88 & 53.55 & s.r.d. & - & & $2^{\circ} 30^{\circ} \mathrm{PM}(\mathrm{E})$ & Bom \\
\hline 89 & $93-61$ & s.r.d. & 10) & a & $\mathrm{C}, 4.0 \mathrm{PM}, 1.020^{\circ} \mathrm{M}$ (D) & Bom \\
\hline 90 & 845 & s.r.d. & G & a & $4 \cap \Gamma \mathrm{P}(\mathrm{F})$ & Bom \\
\hline 91 & 1438 & s.r.d. & 3 & a & 1.0 PN (E) & Bom \\
\hline 92 & 16664 & Basset & 9 & $\mathbf{a}$ & $C(E)$ & Hom \\
\hline 93 & 3346 & Cocker & 7 & a & $3.0^{\circ} 4 \mathrm{PM}^{\circ}$ 1.. $\mathrm{M}$ (I) & $130 \mathrm{~m}$ \\
\hline 94 & 4051 & Basset & 11 & a & 1.० PM ( $\mathrm{F})$ & Hom \\
\hline 95 & 4817 & s.r.d. & 10 & a & 4.० PM (D) & Bom \\
\hline 96 & 4984 & Fox & 9 & $\mathbf{a}$ & 4. $\mathrm{PMI}(\mathrm{D})$ & Bom \\
\hline \multirow[t]{2}{*}{97} & $190-62$ & Miniatura & & & 4.0 PMI (E) & Hom \\
\hline & & Pincher & 9 & $a$ & $1.0 \mathrm{NI}$ & Bom \\
\hline 98 & 1866 & s.r.d. & 12 & $\mathbf{a}$ & 4.0 PM, 1.0 M (E) & Bom \\
\hline 99 & 2806 & Basset & 10 & $\mathbf{a}$ & $4 .^{\circ} \cdot \mathrm{PM}(\mathrm{D})$ & $130 \mathrm{~m}$ \\
\hline 100 & 4482 & s.r.d. & 4 & a & $1 . \circ 2.0^{\circ} 3 .^{\circ} 3 .^{\circ} 4 .^{\circ} \mathrm{PM}$ (D) & 130m \\
\hline
\end{tabular}


Rev. Fac. Med. Vet. S. Paulo - Vol. 8 fasc. 1, 1969

TABELA 1 - (Continuaçāo)

\begin{tabular}{|c|c|c|c|c|c|c|}
\hline Ous- & $\begin{array}{l}\text { Regis- } \\
\text { tro }\end{array}$ & Raca & Idad & & Dentes extraidos & Resultado \\
\hline 101 & $497 s$ & s.r.d. & 10 & a & $40^{\circ} \mathrm{PM}, 10^{\circ} \mathrm{MI}(\mathrm{E})$ & Bom \\
\hline 102 & $279-63$ & s.r.d. & 7 & $\mathbf{a}$ & 1.0 PMI (E) & Bom \\
\hline 103 & 623 & s.r.a. & $8=$ & a & 4. P.I (E) & Bom \\
\hline 104 & 759 & s.r.d. & $5:$ & a & 1.० PM (F) & Bom \\
\hline 105 & 1091 & s.r.d. & 7 & $\mathbf{a}$ & 1. PMI (E) & Bom \\
\hline 106 & 1361 & s.r.d. & 13 & a & 4.0 P.M (D) & Bom \\
\hline 107 & 1515 & s.r.d. & - & & $C(D)$ & Bom \\
\hline 108 & 215i; & s.r.d. & $11=$ & $\mathbf{a}$ & 1.0 PM (E) & Bom \\
\hline 109 & $236 \mathrm{i} 3$ & Conker & $1.1:$ & a & $1.0 \mathrm{PM}$ (D) & $130 \mathrm{~m}$ \\
\hline 110 & 2915 & s.r.d. & $16:$ & a & $1.0 \mathrm{MI}(\mathrm{F})$ & l3om \\
\hline 111 & 3595 & Basset & $11:$ & a & 2." MI (1)) & Bom \\
\hline 112 & 3620 & s.r.d. & 7 & $\mathbf{a}$ & f." PM (E) & Bom \\
\hline 113 & 3713 & s.r.d. & $9:$ & in & $1.0 .21 \quad(1)\}$ & Bom \\
\hline $11 \cdot 4$ & 3959 & s.r.d. & $5:$ & a & $-1.0^{\circ} \mathrm{P} M(\mathrm{D})$ & I3om \\
\hline 115 & 4127 & Pequines & 11 & a & $1.0 \mathrm{MI}(\mathrm{l} \%)$ & Bom \\
\hline 116 & 4127 & I'equines & $11:$ & in & A." PM (E) & Bom \\
\hline 117 & 4519 & s.r.t. & 6; : & $\mathbf{a}$ & II." $P M(E)$ & Bom \\
\hline 118 & 4917 & - & $8:$ & a & $40^{\circ} \mathrm{PM}, 1.020^{\circ}$.II (D) & Bom \\
\hline 119 & $95-6 \cdot 4$ & I3asset & $s:$ & $a$ & 1." PM, 1.0 MI (E) & $130 \mathrm{~m}$ \\
\hline 120 & 1563 & s.r.te & $8=$ & a & A." PMI & $130 \mathrm{~m}$ \\
\hline 121 & 160.4 & s.r.d. & $4:$ & a & 3." PM (D) & $130 \mathrm{~m}$ \\
\hline 122 & 1607 & s.r.d. & $10:$ & $\mathbf{a}$ & $1.02 .03 .0 \mathrm{PMI}$ & Bom \\
\hline 123 & 2048 & 13asset & $12=$ & $\mathrm{a}$ & $\mathrm{C}(\mathrm{H})$ & Bom \\
\hline 124 & 2148 & s.r.d. & 4 & $\mathbf{a}$ & $4.0 \mathrm{PMI}(\mathrm{D})$ & Bom \\
\hline 125 & $2 \times 91$ & s.r.d. & 5 & a & 4.0 l'M (D) & Bom \\
\hline 126 & 3062 & s.r.a. & $10:$ & a & $1.0 \mathrm{IM}(\mathrm{D})$ & 13om \\
\hline 127 & .3586 & s.r.d. & 13 & a & $40^{\circ} \mathrm{PM}(\mathrm{D})$ & $130 \mathrm{~m}$ \\
\hline $12 \mathrm{~s}$ & $36 ;-42$ & s.r.d. & 6 & a & $1.02 .0 \mathrm{PM}(\mathrm{H})$ & Bom \\
\hline 129 & 3776 & s.r.di. & 2 & a & \$.० PMI & Bom \\
\hline 130 & 11124 & Pastor & 8 & a & $3.04 .0 \quad 1 \% M(D)$ & Bom \\
\hline 131 & $17: 39-63$ & Pequinis & $10:$ & a & $3 .^{\circ}+.^{\circ} \mathrm{PMI}(\mathrm{E})$ & Hom \\
\hline 132 & 23149 & s.r.d. & 18 & a & $4.0 \mathrm{PM}(\mathrm{H})$ & I3om \\
\hline 133 & 2609 & s.r.d. & 9 & a & $1.0 P M(F)$ & Bom \\
\hline 137 & 2672 & s.r.d. & 15 & a & $1.0 \mathrm{I}^{2} \mathrm{M}(\mathrm{F})$ & Bom \\
\hline 135 & $414 \mathrm{~s}$ & Pastor & 2 & a & 1.0 PMI (F) & Bom \\
\hline 136 & 5172 & Basset & $14:$ & a & $2.0 \mathrm{MI}(\mathrm{D})$ & Bom \\
\hline 137 & 5172 & Basset & 14 & a & $2 .^{\circ} 3.0 \mathrm{I}(\mathrm{D})$ & Bom \\
\hline 138 & 6305 & Fox & 8 & a & 2.0 PMI (D) & Bom \\
\hline 139 & $37-66$ & s.r.d. & 13 & a & $1.02 .03 .04 .0 \mathrm{PMI}(\mathrm{F})$ & Bom \\
\hline 140 & 219 & s.r.d. & 5 & it & 1.0 PMI (D) & Bom \\
\hline 141 & 3023 & s.r.d. & 12 & a & $30^{\circ} 40^{\circ} \mathrm{PM}, 10^{\circ} \mathrm{M}$ (D) & BOm \\
\hline 1.12 & 3105 & Pequinés & 6 & a & $4.0 \mathrm{PMI}$ (D) & Bom \\
\hline 1.13 & .3218 & Basset & 12 & $\mathbf{a}$ & $20^{\circ} 3.04 .0$ PM. 1.0 .II (D) & 130m \\
\hline 1.44 & 3218 & Bassct & 12 & a & $2 .^{\circ}$ MI (F:) & Bom \\
\hline 1.15 & $33 \times 1$ & s.r.d. & 8 & a & $1.0 \mathrm{PM}(\mathrm{F})$ & Bom \\
\hline 146 & 35.47 & Pequines & - & & $1.0 \mathrm{PM}(\mathrm{F})$ & $\mathrm{Bom}$ \\
\hline 147 & 5161 & c.s. Pastor & 13 & a & $4.0 \mathrm{PM}(\mathrm{D})$ & Bom \\
\hline 148 & 5345 & Pastor & 3 & a & $10^{\circ} \mathrm{PM}(\mathrm{D})$ & Bom \\
\hline 1.19 & 5645 & s.r.d. & 11 & a & $2 .{ }^{\circ} \mathrm{M}$ & $130 \mathrm{~m}$ \\
\hline 150 & 5665 & Polnter & 1 & $\mathbf{a}$ & $40^{\circ} P M(E)$ & 13om \\
\hline 151 & 5972 & Pequines & $5:$ & a & $4.0 \mathrm{PM}\left(\mathrm{F}_{0}\right)$ & Bom \\
\hline 152 & 5972 & Pequines & 5 & $a$ & $4.0 \mathrm{PM}$ (D) & Bom \\
\hline
\end{tabular}

